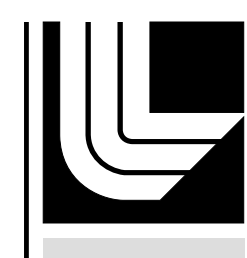

LAWRENCE LIVERMORE NATION A L LABORATORY

Unusual Pulsed X-Ray Emission from the Young, High Magnetic Field Pulsar PSR J1119--6127

M. E. Gonzalez, V. M. Kaspi, F. Camilo, B. M. Gaensler, M. J. Pivovaroff

August 8, 2005

Astrophysics Journal 
This document was prepared as an account of work sponsored by an agency of the United States Government. Neither the United States Government nor the University of California nor any of their employees, makes any warranty, express or implied, or assumes any legal liability or responsibility for the accuracy, completeness, or usefulness of any information, apparatus, product, or process disclosed, or represents that its use would not infringe privately owned rights. Reference herein to any specific commercial product, process, or service by trade name, trademark, manufacturer, or otherwise, does not necessarily constitute or imply its endorsement, recommendation, or favoring by the United States Government or the University of California. The views and opinions of authors expressed herein do not necessarily state or reflect those of the United States Government or the University of California, and shall not be used for advertising or product endorsement purposes. 
Draft Version August 5, 2005

Preprint typeset using ${ }_{\mathrm{LAT}} \mathrm{X}$ style emulateapj v. 2/19/04

\title{
UNUSUAL PULSED X-RAY EMISSION FROM THE YOUNG, HIGH MAGNETIC FIELD PULSAR PSR J1119-6127
}

\author{
M. E. Gonzalez ${ }^{1}$, V. M. Kaspi ${ }^{1}$, F. Camilo ${ }^{2}$, B. M. Gaensler ${ }^{3}$, And M. J. Pivovaroff ${ }^{4}$ \\ Draft version August 5, 2005
}

\begin{abstract}
We present XMM-Newton observations of the radio pulsar PSR J1119-6127, which has an inferred age of 1,700 yr and surface dipole magnetic field strength of $4.1 \times 10^{13} \mathrm{G}$. We report the first detection of pulsed X-ray emission from PSR J1119-6127. In the 0.5-2.0 keV range, the pulse profile shows a narrow peak with a very high pulsed fraction of $(74 \pm 14) \%$. In the $2.0-10.0 \mathrm{keV}$ range, the upper limit for the pulsed fraction is $28 \%$ (99\% confidence). The pulsed emission is well described by a thermal blackbody model with a temperature of $T^{\infty}=2.4_{-0.2}^{+0.3} \times 10^{6} \mathrm{~K}$ and emitting radius of $3.4_{-0.3}^{+1.8} \mathrm{~km}$ (at a distance of $8.4 \mathrm{kpc}$ ). Atmospheric models result in problematic estimates for the distance/emitting area. PSR J1119-6127 is now the radio pulsar with smallest characteristic age from which thermal Xray emission has been detected. The combined temporal and spectral characteristics of this emission are unlike those of other radio pulsars detected at X-ray energies and challenge current models of thermal emission from neutron stars.

Subject headings: ISM: individual (G292.2-0.5) — pulsars: individual (PSR J1119-6127) — supernova remnants - X-rays: ISM
\end{abstract}

\section{INTRODUCTION}

The most commonly studied neutron stars (NSs) are radio pulsars, generally thought to be powered by the loss of rotational kinetic energy due to magnetic braking. The thermal X-rays observed from some pulsars are thought to be emitted at the surface and to originate from initial cooling or from polar caps reheated by magnetospheric processes (see, e.g., Kaspi, Roberts \& Harding 2004, for a review). At low X-ray energies, this thermal emission shows broad pulses of low amplitude. In contrast, highly modulated non-thermal X-rays showing narrow pulses are thought to arise from synchrotron emission in the magnetosphere (e.g., Cheng \& Zhang 1999; Becker \& Aschenbach 2002). Thus, X-ray studies of radio pulsars are an excellent diagnostic of the physics governing NS emission.

PSR J1119-6127 was discovered in the Parkes Multibeam Pulsar Survey (Camilo et al. 2000). It has a spin period $P=0.408 \mathrm{~s} \equiv 1 / \nu$ and period derivative $\dot{P}=$ $4.1 \times 10^{-12}$. The measured braking index for the pulsar of $n=2.91 \pm 0.05\left(\dot{\nu} \propto-\nu^{n}\right)$ implies an upper limit for the age of $1,700 \mathrm{yr}$, making it one of the youngest pulsars known. The pulsar has a spin-down luminosity of $\dot{E}=$ $4 \pi^{2} I \dot{P} / P^{3}=2.3 \times 10^{36} \mathrm{erg} \mathrm{s}^{-1}$ (for a moment of inertia $I \equiv 10^{45} \mathrm{~g} \mathrm{~cm}^{2}$ ) and an inferred surface dipole magnetic field strength of $B \equiv 3.2 \times 10^{19}(P \dot{P})^{1 / 2} \mathrm{G}=4.1 \times 10^{13} \mathrm{G}$. This value of $B$ is among the highest known in the radio pulsar population. PSR J1119-6127 powers a small $\left(3^{\prime \prime} \times 6^{\prime \prime}\right)$ X-ray pulsar wind nebula (PWN, Gonzalez \& Safi-Harb 2003) and lies close to the center of the $15^{\prime}$ -

\footnotetext{
${ }^{1}$ Department of Physics, Rutherford Physics Building, McGill University, Montreal, QC H3A 2T8, Canada.

2 Columbia Astrophysics Laboratory, Columbia University, 550 West 120th Street, New York, NY 10027.

3 Harvard-Smithsonian Centre for Astrophysics, 60 Garden Street, Cambridge, MA 02138.

${ }^{4}$ Lawrence Livermore National Laboratory, P.O. Box 808, L-258, Livermore, CA 94550.
}

diameter supernova remnant (SNR) G292.2-0.5 (Crawford et al. 2001; Pivovaroff et al. 2001). HI absorption measurements imply a kinematic distance for the remnant of $8.4 \pm 0.4 \mathrm{kpc}$ (Caswell et al. 2004), in agreement with estimates made from its location in the Carina spiral arm (Camilo et al. 2000).

Here we report on $X M M-N e w t o n$ observations of PSR J1119-6127 and SNR G292.2-0.5. The X-ray emission from PSR J1119-6127 shows a high-temperature thermal spectrum having a high pulsed fraction at low energies. No pulsations are detected at high energies. These characteristics are unlike those of other radio pulsars detected at X-ray energies. We discuss the possible origins of this emission. A detailed analysis of SNR G292.2-0.5 is deferred to a future paper.

\section{OBSERVATION AND IMAGING ANALYSIS}

SNR G292.2-0.5 and PSR J1119-6127 were observed with XMM-Newton on 2003 June 26. The European Photon Imaging Camera (EPIC) instruments MOS and $\mathrm{PN}$ were operated in full-window and large-window mode, respectively, using the medium filter for both. The temporal resolution was $2.6 \mathrm{~s}$ for MOS and $48 \mathrm{~ms}$ for PN. The data were analysed using the Science Analysis System software (SAS v6.2.0) and standard reduction techniques. The last $5 \mathrm{ks}$ of the PN observation were rejected due to high radiation. The resulting exposure time was $48 \mathrm{ks}$ for MOS1/MOS2 and $43 \mathrm{ks}$ for PN.

Figure 1 shows the combined MOS image of the system in the $0.3-1.5 \mathrm{keV}$ (red), $1.5-3.0 \mathrm{keV}$ (green) and 3.0 $10.0 \mathrm{keV}$ (blue) bands. Individual MOS1/MOS2 images were first binned into pixels of $2 . .5 \times 2.5$ and other point sources in the field were excluded. These images were then added and adaptively smoothed with a Gaussian with $\sigma=5^{\prime \prime}-15^{\prime \prime}$ to obtain signal-to-noise ratios higher than $3 \sigma$. Background images and exposure maps at each energy band were similarly obtained and used to correct the final images. The detailed spatial distribution in the $3.0-10.0 \mathrm{keV}$ (blue) image should be examined with 
caution as this energy band suffered from a high degree of stray-light contamination on XMM-Newton's mirrors from a nearby high-energy source. Here we focus on the bright source at the center of Fig. 1, which has coordinates $\alpha_{2000}=11^{h} 19^{m} 14.65$ and $\delta_{2000}=-61^{\circ} 27^{\prime} 50^{\prime \prime} 2\left(4^{\prime \prime}\right.$ error). This position coincides with the Chandra and radio coordinates of PSR J1119-6127. The spatial resolution of XMM-Newton (half-power diameter of $15^{\prime \prime}$ ) does not allow for the arc-second scale PWN discovered with Chandra to be resolved.

\section{TIMING ANALYSIS}

The PN data were used to search for pulsations from PSR J1119-6127. Examining the raw event list as in Woods et al. (2004) revealed an instrumental timing anomaly that resulted in time tags being shifted forward by $1 \mathrm{~s}$ for events detected after the anomaly took place. We corrected for this by subtracting $1 \mathrm{~s}$ from the original time stamps and from the auxiliary PN file (*AUX.FIT) for the observation. The corrected dataset was then converted to the solar system barycenter.

A circular region of $25^{\prime \prime}$ radius was used to extract the pulsar events. The data were divided into different energy ranges at $0.5-10.0 \mathrm{keV}(620 \pm 30$ photons), $0.5-2.0$ $\mathrm{keV}(340 \pm 25$ photons) and 2.0-10.0 keV (275 \pm 22 photons). From an updated radio ephemeris obtained from regular monitoring at the Parkes radio telescope, the predicted barycentric radio period for the middle of our observation (MJD 52816.54) was $2.44972914 \mathrm{~Hz}$. The $Z_{n}^{2}$ test (Buccheri et al. 1983) with harmonics $n=\{1,2,4,8\}$ was used to search for pulsations. The most significant signal was detected in the $0.5-2.0 \mathrm{keV}$ range with $Z_{2}^{2}=$ $52.8(6.6 \sigma$ significance) at a frequency of $2.449726(6) \mathrm{Hz}$ ( $1 \sigma$ errors). This frequency is in agreement with the radio prediction for PSR J1119-6127. In the $2.0-10.0 \mathrm{keV}$ range, no signal was found with a significance $>2.8 \sigma$. In the $0.5-10.0 \mathrm{keV}$ range, the above signal was detected with a $5.2 \sigma$ significance.

Figure 2 shows the resulting pulse profiles at $0.5-2.0$ $\mathrm{keV}$ (top, left) and 2.0-10.0 keV (top, right). The background was estimated from a nearby region away from bright SNR knots. The horizontal dashed lines represent our estimates for the contribution from the pulsar's surroundings (see $\S 5.1$ ). The resulting pulsed fraction $\left[\mathrm{PF} \equiv\left(F_{\max }-F_{\min }\right) /\left(F_{\max }+F_{\min }\right)\right]$ is labeled in Fig. 2 ( $1 \sigma$ statistical errors). In the $2.0-10.0 \mathrm{keV}$ range, we derive an upper limit for the pulsed fraction of $28 \%$ (at the $99 \%$ confidence level). For this, we used the maximum power obtained from a Fourier analysis of the data for a small range of frequencies centered on the radio prediction (within our errors) and assumed a worse-case sinusoidal profile. An upper limit for the pulse amplitude can then be derived by calculating an upper limit on the power that could still be present above the observed maximum at a specific level of confidence. For a detailed description of this method we refer to Vaughan et al. (1994), and e.g., Ransom et al. (2002).

The pulse profile in the $0.5-2.0 \mathrm{keV}$ range shows a single, narrow pulse with a high $\mathrm{PF}=(74 \pm 14) \%$. We then modeled the profile with a 1-D gaussian using Sherpa (v.3.2.0). The best-fit value for the full-width at half maximum was $0.26_{-0.06}^{+0.08} P$ ( $1 \sigma$ errors) with $\chi^{2}$ (dof) $=$ 2.1(4) and a probability of 0.72. A sinusoidal model re- sulted in $\chi^{2}($ dof $)=8.9(5)$ with a probability of 0.11 . Due to the limited statistics available, the gaussian fit is preferred with a probability of only $2.3 \times 10^{-2}$ (according to the $F$-test). Additional X-ray observations at higher resolution are needed to further constrain the pulse shape.

Correlating the times of arrival for the radio pulse at $1.4 \mathrm{GHz}$ with the $\mathrm{X}$-ray pulse, we find that the radio peak arrives $26 \mathrm{~ms}$ before the centre of the X-ray peak (with $\sim 3 \mathrm{~ms}$ uncertainty). This corresponds to phase 0 in the pulse profiles of Fig. 2. Taking into account the low temporal resolution of the $X M M-N e w t o n$ observation (phase bin width of $51 \mathrm{~ms}$ ), this result suggests that the radio peak is in phase with the X-ray peak within our uncertainties, or possibly just slightly ahead. In addition, although it is unlikely that the EPIC-PN timing anomaly present during the observation affected the absolute timing (XMM-Newton HelpDesk, private communication), we regard this result with caution until it can be confirmed.

\section{SPECTRAL ANALYSIS}

The EPIC data were used to perform a spectral analysis of PSR J1119-6127. Circular regions with radii of $20^{\prime \prime}$ and $25^{\prime \prime}$ were used for MOS and PN, encompassing $\sim 75 \%$ and $\sim 78 \%$ of the source photons, respectively. The derived fluxes have been corrected accordingly. Background regions were chosen from nearby areas away from bright SNR knots. The spectra were fit in the $0.5-10.0$ $\mathrm{keV}$ range using XSPEC (v.11.3.0) with a minimum of 20 counts per bin from a total of $240 \pm 19,210 \pm 18$, and $620 \pm 30$ background-subtracted counts in MOS1, MOS2 and $\mathrm{PN}$, respectively.

One-component models were first fit to the phaseaveraged MOS and PN data. A power-law model provided a better fit than a thermal blackbody model (BB), giving $\chi^{2}($ dof $)=113(68)$ and $\chi^{2}($ dof $)=180(68)$, respectively. However, it was evident from the fit residuals and the improvement in $\chi^{2}$ that two-component models were needed in order to describe the low and high energy portions of the spectra. The derived fits from twocomponent models are summarised in Table 1. In these fits, a non-thermal power-law component with photon index $\Gamma \sim 1.5$ described the high-energy emission in the spectra well. In turn, various models were used to describe the low-energy emission. A magnetised hydrogen atmosphere model (Atm; Zavlin et al. 1996) required a small distance of $\lesssim 2 \mathrm{kpc}$ to account for the observed emission (or conversely an implausibly large emitting radius of $27_{-2}^{+4} \mathrm{~km}$ at $8.4 \mathrm{kpc}, 1 \sigma$ range). Such a small distance can be ruled out from $\mathrm{HI}$ absorption measurements (Caswell et al. 2004). Currently available (non-magnetic) atmosphere models of higher metallicity (Gänsicke et al. 2002) were also used and found to produce poor fits to the observed emission. At a fixed distance of $8.4 \mathrm{kpc}$, the derived temperatures were $\sim 1.9 \mathrm{MK}\left[\chi^{2}(\right.$ dof $\left.)=85(67)\right]$ and $<1.2 \mathrm{MK}\left[\chi^{2}(\operatorname{dof})=114(67)\right]$ for solar and pure iron compositions, respectively. However, when the distance was allowed to be a free parameter, the models converged to unrealistic values (e.g., $<200$ pc for temperatures $<1.4 \mathrm{MK}$ ) with no improvement on the resulting $\chi^{2}$.

We also extracted PN spectra from the "pulsed" and "unpulsed" regions of the pulse profile, at phases 0.7-

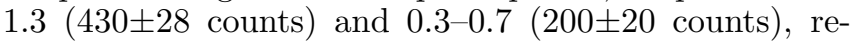


spectively. These spectra are shown in Figure 2 (bottom) and were well fit by two-component models that agree with those derived for the phase-averaged spectra. For example, a blackbody plus power-law model yielded $T^{\infty}=2.8 \pm 0.4 \mathrm{MK}$ and $\Gamma=1.4_{-0.2}^{+0.5}(1 \sigma$ errors $)$. The main difference between the pulsed and unpulsed spectra was found to be the relative contributions of the model components. The pulsed spectrum is dominated by the soft component below $2 \mathrm{keV}$, where it contributes $\sim 85 \%$ $(\sim 90 \%)$ of the absorbed (unabsorbed) flux. In turn, the unpulsed spectrum is dominated by the hard, power-law component even below $2 \mathrm{keV}$, where it contributes $\sim 65 \%$ ( $\sim 55 \%)$ of the absorbed (unabsorbed) flux. For both pulsed and unpulsed spectra, the power-law component contributes $>90 \%$ of the flux above $2 \mathrm{keV}$ (either absorbed or unabsorbed).

\section{DISCUSSION}

\subsection{Emission Characteristics}

The XMM-Newton observation of PSR J1119-6127 allowed us to detect, for the first time, X-ray pulsations from this high-magnetic-field pulsar. The pulsations are only detected in the $0.5-2.0 \mathrm{keV}$ range with a single, narrow peak and a very high pulsed fraction of $(74 \pm 14) \%$.

The emission detected within the XMM-Newton extraction regions is a combination of pulsar, PWN and diffuse SNR emission (see Fig. 1). In addition, the derived spectra from these regions are well fit by twocomponent models. We then argue that the hard spectral component detected arises primarily from the pulsar's surroundings and not from the NS itself. Using a high-resolution archival Chandra observation of the field (see Gonzalez \& Safi-Harb 2003), we estimate that the PWN plus SNR emission within a $25^{\prime \prime}$ radius, excluding the pulsar, is well described by a power-law model with $\Gamma=1.8_{-0.6}^{+0.8}$ and unabsorbed flux in the $0.5-10.0 \mathrm{keV}$ range of $0.9_{-0.5}^{+0.6} \times 10^{-13} \mathrm{erg} \mathrm{s}^{-1} \mathrm{~cm}^{-2}(1 \sigma$ errors $)$. These values are in good agreement with the hard, power-law component shown in Table 1. Although a small contribution from the pulsar to this hard emission cannot be ruled out, it would not affect our results on the pulsar's soft emission.

The soft component detected with $X M M-N e w t o n$ must then arise from PSR J1119-6127, as is further supported by the pulsed emission having a distinctly soft spectrum (see $\S 4$ ). Such emission is unlikely to have a non-thermal origin. Non-thermal X-rays from radio pulsars have photon indices in the range $0.5<\Gamma<2.7$ (e.g., Gotthelf 2003). Synchrotron emission models due to accelerated particles in the magnetosphere predict $\Gamma \lesssim 2$ (e.g., Cheng \& Zhang 1999; Rudak \& Dyks 1999). The soft component of the pulsed emission from PSR J1119-6127, if interpreted as non-thermal, has a steeper spectrum $(\Gamma=6.5 \pm 0.9)$ than those observed or predicted and would imply an acceleration mechanism that cannot be achieved with current models.

The derived blackbody temperature for PSR J1119 6127 of $T_{b b}^{\infty}=2.4_{-0.2}^{+0.3} \mathrm{MK}$ is among the highest seen for radio pulsars. Although it is similar to those found in a few other pulsars, none of them exhibits a higher temperature at statistically significant levels (e.g, PSR J0218+4232 has a characteristic age of $\tau_{c}=P / 2 \dot{P}=$ $5.1 \times 10^{8}$ yr and a blackbody temperature of $T_{b b}^{\infty}=$
2.9 \pm 0.4 MK; Webb et al. 2004). In addition, PSR J11196127 is now the youngest radio pulsar from which thermal emission has been detected, the next youngest being Vela $\left(\tau_{c}=11 \mathrm{kyr}\right)$ with a blackbody temperature $T_{b b}^{\infty}=$ $1.47 \pm 0.06 \mathrm{MK}$ (Pavlov et al. 2001). For comparison, the young Crab pulsar $\left(\tau_{c}=1.3 \mathrm{kyr}\right)$ and PSR J0205+6449 $\left(\tau_{c}=5.4 \mathrm{kyr}\right.$, in the SNR 3C 58) have upper limits on the possible blackbody emission from their entire surface of $T_{b b}^{\infty}<1.97 \mathrm{MK}$ (Weisskopf et al. 2004) and $T_{b b}^{\infty}<1.02 \mathrm{MK}$ (Slane et al. 2004), respectively, at the $3 \sigma$ confidence level.

The observed pulsed fraction for the thermal emission in PSR J1119-6127 is also higher than those of other radio pulsars. Sources for which the thermal emission arises from the entire surface or from localized regions show pulsed fractions at low energies of $<42 \%$ (e.g., Pavlov et al. 2001, 2002; Becker \& Aschenbach 2002). The narrow peak in the pulse profile also appears to differ from the broad pulsations seen in thermal emission from radio pulsars at low energies. Although a multi-component pulse profile (e.g., sine curve plus a narrow peak) in the 0.5-2.0 keV range cannot be ruled out for PSR J1119 6127 and requires additional observations, we expect any pulse components to show a soft spectrum consistent with the $3 \sigma$ values in Table 1 and the narrow component to exhibit a high $\mathrm{PF}>50 \%$.

\subsection{Thermal Emission Mechanisms}

We first consider the observed characteristics in light of conventional models for NS emission. Thermal emission from polar-cap reheating, due to return currents from the "outer gap" (Cheng \& Zhang 1999) or from close to the polar cap (Harding \& Muslimov 2001), is expected to have an X-ray luminosity of $\lesssim 10^{-5} \dot{E}$ in young sources. This is at least 2 orders of magnitude below our value for the luminosity of $0.9_{-0.2}^{+1.1} \times 10^{-3} \dot{E}$ (see Table $1, \mathrm{BB}+\mathrm{PL}$ model). In addition, although polar-cap reheating has been proposed to explain similar characteristics to that of PSR J1119-6127 in some older pulsars $\left(\tau_{c} \gtrsim 1 \mathrm{Myr}\right.$, e.g., Becker \& Aschenbach 2002), such emission implies much smaller emitting areas (radius $<1 \mathrm{~km}$, see e.g., Webb et al. 2004).

Thermal emission may also arise from the entire surface of the NS due to initial cooling. While the observed blackbody luminosity is in line with predictions from cooling models, the observed blackbody temperature is slightly higher than predicted $(\lesssim 1.8 \mathrm{MK}$, e.g., Yakovlev et al. 2004) and the blackbody radius of $R_{b b}^{\infty}$ $=3.4_{-0.9}^{+4.3} d_{8.4} \mathrm{~km}$ ( $3 \sigma$ errors) is smaller than allowed from NS equations of state $\left(R^{\infty} \gtrsim 12 \mathrm{~km}\right.$, Lattimer \& Prakash 2000). The surface temperature can be made more consistent with those expected from initial cooling if a hydrogen atmosphere model is used (see Table 1, and e.g., Pavlov et al. 2002). However, a recent study argues that hydrogen cannot be maintained long-term in atmospheres of NSs having magnetic fields as high as that of PSR J1119-6127 due to diffusive nuclear burning, with only heavier elements surviving long-term (Chang et al. 2004). Regardless of this, atmospheric models result in high $\chi^{2}$ values and/or problematic estimates for the distance/emitting area (see $\S 4$ ).

If the thermal emission originates from the entire surface due to initial cooling, the pulse profile and pulsed 
fraction in particular are highly problematic, as low amplitude, broad, sinusoidal profiles are expected (e.g., Becker \& Aschenbach 2002; Pavlov et al. 2002). The surface brightness distribution on a highly magnetic star cannot produce pulsed fractions higher than $37 \%$ due to General Relativistic light bending. This applies for all reasonable NS compactnesses $(\propto M / R$, the mass-toradius ratio of the star), extreme viewing geometries and large beaming of the emergent radiation (DeDeo et al. 2001).

Larger pulsed fractions can be obtained if the emission arises from localized hot spots on a highly magnetised atmosphere. However, the possible physical mechanisms leading to this geometry are not well understood and has led to suggestions of, e.g., metallicity gradients on the surface due to magnetically channelled fallback material (Pavlov et al. 2000) or nonuniform heating of the atmosphere due to magnetic field decay (Thompson \& Duncan 1996). In any case, previous work suggests that if two (isotropic) antipodal hot spots are present, the large pulsed fraction in PSR J1119-6127 can only be achieved for extreme viewing geometries, extremely small or large NS compactnesses, and small spot sizes (Psaltis et al. 2000; Özel et al. 2001). These contraints are relaxed if a single hot spot is present on the surface, although it is particularly uncertain how such a geometry could be physically achieved; perhaps the NS has an off-centre magnetic dipole. Regardless of this, accounting for the higher effective temperature in PSR J1119-6127 than in other young pulsars is still an issue.

Currently, modelling of the resulting pulsed fraction from hot spots using appropriate atmospheric parameters for the case of PSR J1119-6127 is underway (W. Ho \& P. Chang, private communication). Preliminary results suggest that improved atmospheric models and including effects from limb darkening due to magnetic fields can reproduce the observed pulsed fraction over a wider range of parameters for hot spot emission. However, broad pulsations are expected in this case and the physical origin for such spots is still uncertain.

Thus, understanding the thermal emission from PSR J1119-6127 in light of conventional NS emission mechanisms requires further theoretical studies to be made. Although the particular reason for such emission is not clear at present, it is likely to be related to the high inferred magnetic field, an unusual surface temperature distribution and/or an unusual beaming from the surface. The pulsar's radio emission reveals nothing out of the ordinary that would hint at such peculiar X-ray properties. The X-ray characteristics distinguish PSR J1119-6127 from other radio pulsars detected at X-ray energies, even from those with similar spin characteristics (of which there are only a few). For example, PSR J1846-0258 in the SNR Kes 75 has almost identical spin properties $\left(P=0.324 \mathrm{sec}, B=4.8 \times 10^{13} \mathrm{G}\right.$ and age $\lesssim 1,000-1,700$ yr). However, it is a bright $\mathrm{X}$-ray source with a standard power-law spectrum with $\Gamma=1.39 \pm 0.04$ and broad pulse profile (Helfand et al. 2003; Mereghetti et al. 2002).
The emission observed from PSR J1119-6127 also allows us to consider alternate models from those of radio pulsars. For example, it has been proposed that some (and maybe all) neutron stars are quark stars (Witten 1984), which are generally thought to cool faster than NSs or become indistinguishable from them after an initial fast cooling period (Weber 2004). Quark stars can be kept hotter for longer by including the effects of $e^{+} e^{-}$ pair formation from the surface, although the observed emission would harden and deviate largely from a blackbody (Page \& Usov 2002). This does not appear to be the case for PSR J1119-6127 as its spectrum is well described as a blackbody. Newly discovered photon emission processes are also expected to keep the star hot and conserve the blackbody spectrum (Vogt et al. 2004), but detailed models of the cooling history in this case are not currently available.

Unlike radio pulsars, the observed emission from "magnetars" is thought to be powered by the decay of an ultrastrong magnetic field $\left(10^{14-15} \mathrm{G}\right.$, Thompson \& Duncan 1995). The high thermal temperature and high pulsed fraction for PSR J1119-6127 is reminiscent of magnetar emission (see, e.g., Woods \& Thompson 2004, for a review). The high pulsed fraction and observed pulse profile for PSR J1119-6127, as well as for the Anomalous Xray Pulsars, are consistent with magnetar models where the emission arises from a single hot spot on the surface (Özel et al. 2001; Özel 2002), although, again, the reason for this geometry is unclear. These models also predict little dependence of the pulsed fraction with energy, which can be tested in PSR J1119-6127 with additional observations. However, unlike PSR J1119-6127, magnetars show a significant (pulsed) power-law contribution in their spectra, have X-ray luminosities much higher than the available rotational energy and, thus far, have not shown radio emission. To date, no other apparently high magnetic field radio pulsar has shown emission characteristics similar to that of magnetars (e.g., PSR J18141744, Pivovaroff et al. 2000; PSR J1846-0258, Helfand et al. 2003; PSR J1718-3718, Kaspi \& McLaughlin 2004), although it has been suggested that they represent magnetars in quiescence (Kaspi \& McLaughlin 2004).

We thank A. Cumming, A. Harding, R. Ouyed, M. Roberts, L. Bildstein, D. Lai, W. Ho, P. Chang and R. Rutledge for useful discussions. This work was supported by an NSERC Research Grant and NASA grant NAG513572. VMK is a Canada Research Chair and Steacie Fellow and received support from an NSERC Discovery Grant and Steacie Supplement, from FQRNT and CIAR. Radio timing data for PSR J1119-6127 were collected at the Parkes telescope, a part of the Australia Telescope which is funded by the Commonwealth of Australia for operation as a National Facility managed by CSIRO. We thank the members of the multibeam survey team, in particular R. Manchester, for help with collecting these data.

\section{REFERENCES}

Becker, W., \& Aschenbach, B. 2002, in Proc. 270th WE-Heraeus Seminar on Neutron Stars, Pulsars, and Supernova Remnants, ed. W. Becker, H. Lesch, \& J. Trümper (MPE Rep. 278; Garching: MPE), 64 (astro-ph/0208466)
Buccheri, R., et al. 1983, A\&A, 128, 245

Camilo, F., et al. 2000, ApJ, 541, 367 
TABLE 1

Fits to THE $X M M-N e w t o n$ PHASE-AVERAGED SPECTRUM OF PSR J1119-6127

\begin{tabular}{|c|c|c|c|}
\hline Parameter & $\begin{array}{c}\mathrm{PL}+\mathrm{PL} \\
( \pm 1 \sigma)\end{array}$ & $\begin{array}{l}\mathrm{BB}+\mathrm{PL} \\
( \pm 1 \sigma)\end{array}$ & $\begin{array}{l}\mathrm{Atm}^{\mathrm{a}}+\mathrm{PL} \\
( \pm 1 \sigma)\end{array}$ \\
\hline$N_{H}\left(10^{22} \mathrm{~cm}^{-2}\right)$ & $2.3_{-0.3}^{+0.4}$ & $1.6_{-0.3}^{+0.4}$ & $1.9_{-0.3}^{+0.5}$ \\
\hline$\chi^{2}(\mathrm{dof})$ & $79(66)$ & $78(66)$ & $78(66)$ \\
\hline & \multicolumn{3}{|c|}{ Soft component characteristics } \\
\hline$\Gamma$ or $T^{\infty}$ & $6.5 \pm 0.9$ & $2.4_{-0.2}^{+0.3} \mathrm{MK}$ & $0.9 \pm 0.2 \mathrm{MK}$ \\
\hline$R^{\infty}(\mathrm{km})$ & & $3.4_{-0.3}^{+1.8}$ & 12 (fixed) \\
\hline$d(\mathrm{kpc})$ & 8.4 (fixed) & 8.4 (fixed) & $1.6_{-0.9}^{+0.2}$ \\
\hline$f_{a b s} \mathrm{~b}\left(10^{-14}\right)$ & $2.1_{-0.9}^{+2.3}$ & $1.5_{-0.2}^{+1.8}$ & $1.7_{-0.4}^{+7.0}$ \\
\hline$f_{\text {unabs }} \mathrm{b}\left(10^{-13}\right)$ & $63_{-32}^{+57}$ & $2.4_{-0.5}^{+3.0}$ & $7.2_{-1.6}^{+31}$ \\
\hline$L_{X}{ }^{\mathrm{b}}\left(10^{33}\right)$ & $53_{-27}^{+50}$ & $2.0_{-0.4}^{+2.5}$ & $0.22_{-0.05}^{+0.88}$ \\
\hline & \multicolumn{3}{|c|}{ Hard component characteristics } \\
\hline$\Gamma$ & $1.3_{-0.2}^{+0.5}$ & $1.5_{-0.2}^{+0.3}$ & $1.5_{-0.3}^{+0.2}$ \\
\hline$f_{a b s}{ }^{\mathrm{b}}\left(10^{-14}\right)$ & $7.1_{-1.5}^{+10}$ & $7.4_{-1.0}^{+3.6}$ & $7.3_{-2.7}^{+4.7}$ \\
\hline$f_{\text {unabs }} \mathrm{b}\left(10^{-13}\right)$ & $1.0_{-0.2}^{+1.6}$ & $1.1_{-0.2}^{+0.6}$ & $1.1_{-0.3}^{+0.8}$ \\
\hline$L_{X}{ }^{\mathrm{b}}\left(10^{33}\right)$ & $0.8_{-0.2}^{+1.3}$ & $0.9_{-0.1}^{+0.5}$ & $0.04 \pm 0.02$ \\
\hline
\end{tabular}

a The atmospheric model was computed with $B=10^{13} \mathrm{G}$ and pure hydrogen composition. The local values for the temperature, $T$, and radius, $R=10 \mathrm{~km}$, of the star have been redshifted to infinity according to $T^{\infty}=T\left(1-2 G M / R c^{2}\right)^{1 / 2}$ and $R^{\infty}=$ $R\left(1-2 G M / R c^{2}\right)^{-1 / 2}$, with $M=1.4 M_{\odot}$.

$\mathrm{b}$ The $0.5-10.0 \mathrm{keV}$ absorbed and unabsorbed fluxes, $f_{a b s}$ and $f_{\text {unabs }}$, have units of ergs s ${ }^{-1} \mathrm{~cm}^{-2}$. The $0.5-10.0 \mathrm{keV} \mathrm{X-ray}$ luminosity, $L_{X}$, at the distance $d$, is in units of ergs s $\mathrm{s}^{-1}$.

Caswell, J. L., McClure-Griffiths, N. M., \& Cheung, M. C. M. 2004, MNRAS, 352, 1405

Chang, P., Arras, P., \& Bildsten, L. 2004, ApJ, 616, L147

Cheng, K. S., \& Zhang, L. 1999, ApJ, 515, 337

Crawford, F., et al. 2001, ApJ, 554, 152

DeDeo, S., Psaltis, D., \& Narayan, R. 2001, ApJ, 559, 346

Gänsicke, B. T., Braje, T. M., \& Romani, R. W. 2002, A\&A, 386, 1001

Gonzalez, M., \& Safi-Harb, S. 2003, ApJ, 591, L143

Gotthelf, E. V. 2003, ApJ, 591, 361

Harding, A. K., \& Muslimov, A. G. 2001, ApJ, 556, 987

Helfand, D. J., Collins, B. F., \& Gotthelf, E. V. 2003, ApJ, 582, 783

Kaspi, V. M., \& McLaughlin, M. A. 2004, ApJ, in press

Lattimer, J. M., \& Prakash, M. 2000, PhR, 333, 121

Mereghetti, S., Bandiera, R., Bocchino, F., \& Israel, G. L. 2002, ApJ, 574, 873

Özel, F., Psaltis, D., \& Kaspi, V. M. 2001, ApJ, 563, 255

Özel, F. 2002, ApJ, 575, 397

Page, D., \& Usov, V. V. 2002, Phys. Rev. Lett., 89, 131101

Pavlov, G. G., et al. 2000, ApJ, 531, L53

Pavlov, G. G., Zavlin, V. E., \& Sanwal, D. 2002, in Proc. 270th WE-Heraeus Seminar on Neutron Stars, Pulsars, and Supernova Remnants, ed. W. Becker, H. Lesch, \& J. Trümper (MPE Rep. 278; Garching: MPE), 273 (astro-ph/0206024)

Pavlov, G. G., et al. 2001, ApJ, 552, L129
Pivovaroff, M. J., et al. 2001, ApJ, 554, 161

Psaltis, D., Özel, F., \& DeDeo, S. 2000, ApJ, 544, 390

Ransom, S. M., Eikenberry, S. S., \& Middleditch, J. 2002, AJ, 124, 1788

Rudak, B., \& Dyks, J. 1999, MNRAS, 303, 477

Slane, P., Helfand, D. J., van der Swaluw, E., \& Murray, S. S. 2004, ApJ, 616, 403

Thompson, C., \& Duncan, R. C. 1995, MNRAS, 275, 255

Thompson, C., \& Duncan, R. C. 1996, ApJ, 473, 322

Vaughan, B. A., et al. 1994, ApJ, 435, 362

Vogt, C., Rapp, R., \& Ouyed, R. 2004, Nuclear Physics A, 735, 543

Webb, N. A., Olive, J.-F., \& Barret, D. 2004, A\&A, 417, 181

Weber, F. 2004, To appear in "Progress in Particle and Nuclear Physics". (astro-ph/0407155).

Weisskopf, M. C., et al. 2004, ApJ, 601, 1050

Witten, E. 1984, Phys. Rev. D, 30, 272

Woods, P. M., et al. 2004, ApJ, 605, 378

Woods, P. M., \& Thompson, C. 2004, in Compact Stellar X-ray Sources, ed. W. H. G. Lewin \& M. van der Klis (UK: Cambridge University Press), in press (astro-ph/0406133)

Yakovlev, D. G., et al. 2004, Advances in Space Research, 33, 523

Zavlin, V. E., Pavlov, G. G., \& Shibanov, Y. A. 1996, A\&A, 315, 141

This work was performed under the auspices of the U.S. Department of Energy by University of California, Lawrence Livermore National Laboratory under contract W-7405-Eng-48. 


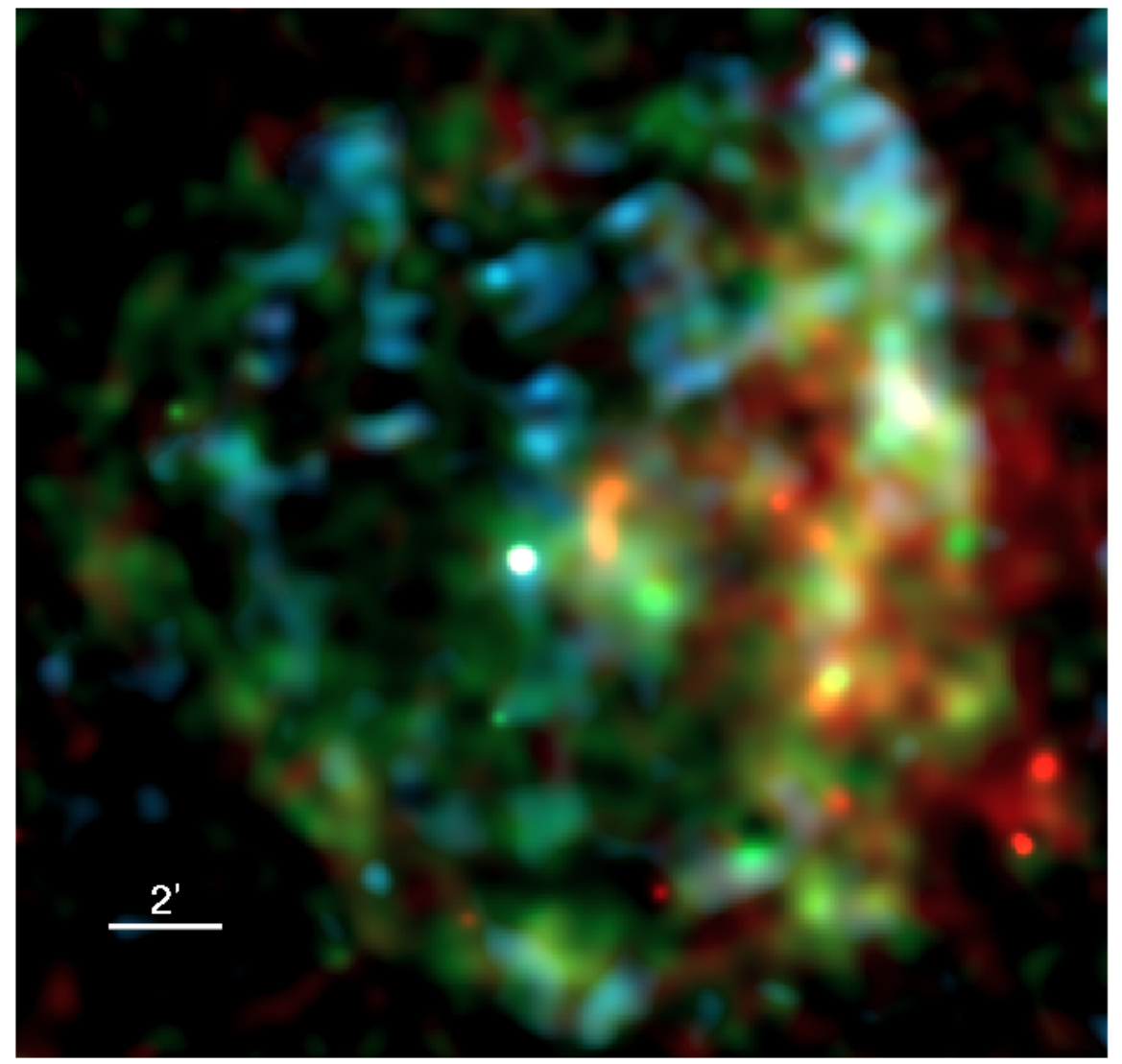

FIG. 1.- EPIC-MOS combined image of SNR G292.2-0.5 and PSR J1119-6127 $\left(19^{\prime} \times 19^{\prime}\right.$ field) in the 0.3-1.5 keV (red), 1.5-3.0 keV (green) and 3.0-10.0 keV (blue) ranges. The image reveals for the first time the detailed morphology of the remnant at X-ray energies. The large east-west asymmetry at low energies has been attributed to the presence of a molecular cloud on the east side of the field (Pivovaroff et al. 2001). 

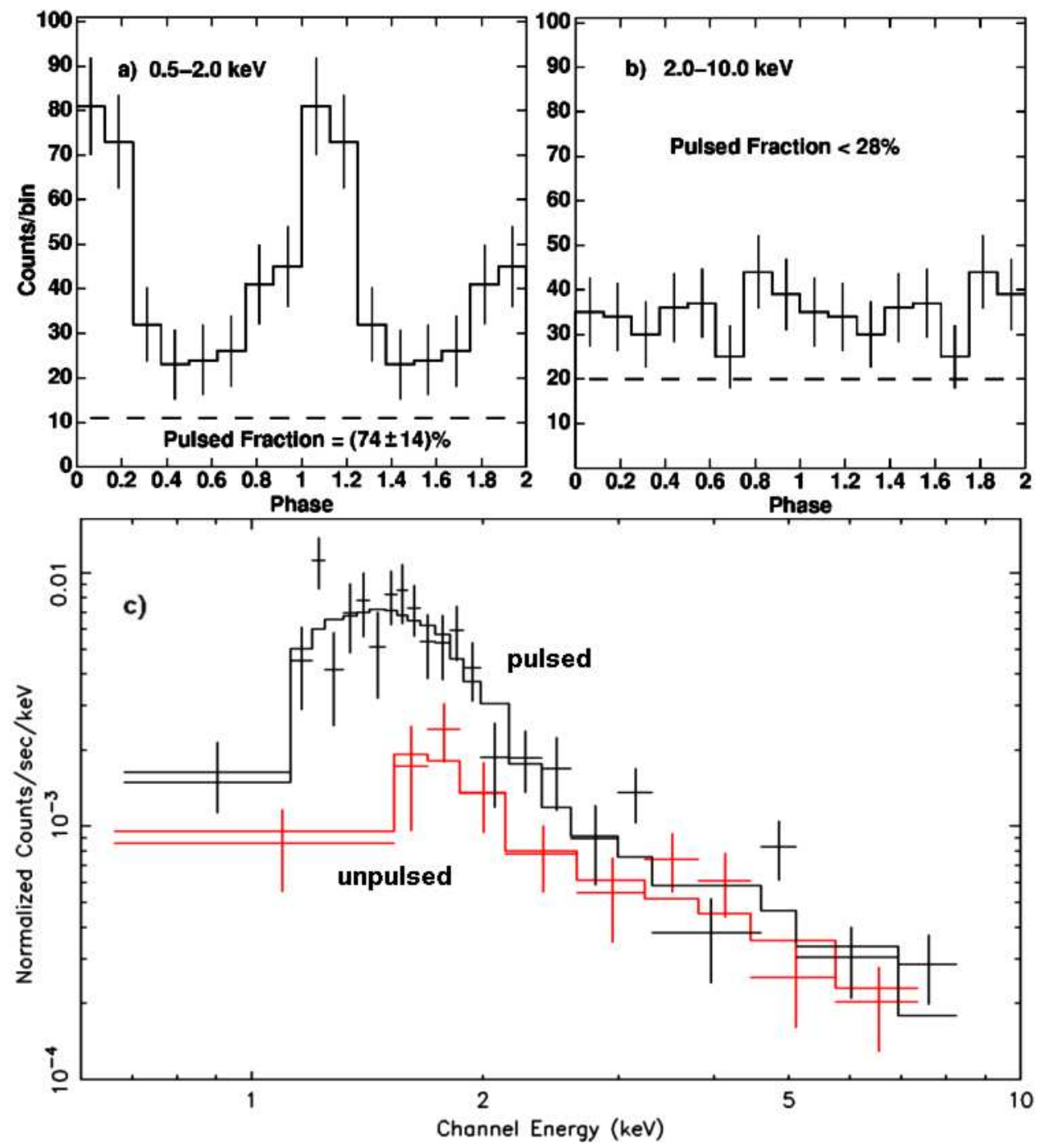

FIG. 2.- Top: X-ray pulse profiles of PSR J1119-6127 in the $0.5-2.0 \mathrm{keV}$ (left) and 2.0-10.0 keV (right) ranges. Errors bars are $1 \sigma$ and two cycles are shown. The peak of the radio pulse is at phase 0 . The dashed lines represent our estimates for the contribution from the pulsar's surroundings (see §5.1). Bottom: EPIC-PN spectra obtained for the pulsed (black) and unpulsed (red) regions of the pulse profile with their respective best-fit blackbody plus power-law model (solid curves). [See the electronic version of the Journal for a color version of this figure.] 